\title{
Unexpected death from a colloid cyst
}

\author{
Christian Hohenstein • Steffen Herdtle
}

Received: 17 August 2009 / Accepted: 3 December 2009 /Published online: 27 February 2010

(C) Springer-Verlag London Ltd 2010

\begin{abstract}
Background Colloid cysts are usually benign brain tumors, which rarely cause acute neurological deterioration with sudden death due to an acute increase of intracranial pressure. So far, the final pathophysiology and clinical signs of impending death are unclear in this context.

Aim We present a case of an adolescent who presented with symptoms similar to gastroenteritis.

Results He unexpectedly developed a generalized seizure, acute pulmonary edema and life-threatening cardiac dysrhythmia.

Conclusion Subtle distinctions between symptoms due to intracranial hypertension, which typically cause headache and vomiting, and true gastroenteritis are discussed as well as the pathophysiology of neurogenic pulmonary edema and the origin of cerebral-triggered cardiac dysrhythmias.
\end{abstract}

Keywords Unexpected death · Colloid cyst .

Cardiopulmonary complication

Electronic supplementary material The online version of this article (doi:10.1007/s12245-009-0153-4) contains supplementary material, which is available to authorized users.

C. Hohenstein $(\triangle)$

Department of Emergency Medicine, University Hospital Jena,

Erlanger Allee 101,

07740 Jena, Germany

e-mail: christian.hohenstein@med.uni-jena.de

S. Herdtle

Department of Anaesthesiology, Hospital Nuremberg North,

Prof.-Ernst-Nathan-Str. 1,

90419 Nuremberg, Germany

\section{Patient presentation}

A previously healthy adolescent male presented with a 3-day history of headache and vomiting, initially diagnosed as gastroenteritis. On the day of admission, he presented with slightly lethargy, but was able to communicate and follow simple commands. Unexpectedly, he developed a generalized seizure and different types of unstable tachycardia. Multiple cardioversions and amiodarone finally resulted in sinus tachycardia. Sudden pulmonary edema required intubation, and a few minutes later, the pupils became dilated and fixed. An immediate $\mathrm{CT}$ scan showed a hyperdense colloid cyst in the foramen of Monro with massive hydrocephalus. A chest x-ray showed a picture of a white lung similar to ARDS. A bilateral ventricular external shunt performed by neurosurgeons was of no avail; the patient died after 2 days decerebrated. No post-mortem examination was performed.

\section{Discussion}

Third ventricular colloid cysts can affect young patients and often present with mild symptoms [1]. Diagnosed in a timely manner, these ependymomas can easily be removed by neurosurgeons. A delay in diagnosis can lead to an acute clinical deterioration with unexpected and sudden death [2-4]. The diagnosis of a viral illness is the typical pitfall. Cardiac and pulmonary complications can accompany brain herniation and again distract physicians from the underlying cerebral origin [5-8].

Colloid cysts, although rare, should remain in the differential diagnosis in young patients with headache and vomiting - especially if no further symptoms are present. Immediate diagnostic testing in suscpected cases is essen- 
tial since an acute hydrocephalus can be disastrous within minutes. There was no positive family history in this case, but familial clusters have been described [9-11].

\section{References}

1. Elgamal EA, Richards PG (2006) Sudden death in children due to intracranial mass lesion. Childs Nerv Syst 22(3):305-309

2. Humphries RL, Stone CK, Bowers RC (2008) Colloid cyst: A case report and literature review of a rare but deadly condition. $\mathrm{J}$ Emerg Med

3. Opeskin K, Anderson RM (1995) Colloid cysts of the third ventricle: fatal outcomes associated with unusual presentation. J Clin Neurosci 2(4):307-311

4. Shemie S et al (1997) Acute obstructive hydrocephalus and sudden death in children. Ann Emerg Med 29(4):524-528
5. Baumann A et al (2007) Neurogenic pulmonary edema. Acta Anaesthesiol Scand 51(4):447-455

6. Bunai Y et al (2008) Sudden death due to undiagnosed intracranial hemangiopericytoma. Am J Forensic Med Pathol 29 (2): $170-172$

7. Grosse-Wortmann L, Bindl L, Seghaye MC (2006) Multiple types of cardiac arrhythmias in a child with head injury and raised intracranial pressure. Pediatr Cardiol 27(2):286-288

8. Sedy J et al (2008) Mechanisms of neurogenic pulmonary edema development. Physiol Res 57(4):499-506

9. Akins PT et al (1996) Familial colloid cyst of the third ventricle: case report and review of associated conditions. Neurosurgery 38 (2):392-395

10. Socin HV et al (2002) Familial colloid cyst of the third ventricle: neuroendocrinological follow-up and review of the literature. Clin Neurol Neurosurg 104(4):367-370

11. Stoodley MA, Nguyen TP, Robbins P (1999) Familial fatal and near-fatal third ventricle colloid cysts. Aust NZ J Surg 69 (10):733-736 